\title{
Kinematical Relativistic Corrections for Earth's Rotation Parameters
}

\author{
V.A. Brumberg \\ Institute of Applied Astronomy, 191187 St. Petersburg, Russia \\ Pierre Bretagnon \\ Institut de mécanique céleste, 75014 Paris, France
}

\begin{abstract}
Dynamical theories of the Earth's rotation like SMART97 (Bretagnon et al., 1998) are to be considered in a DGRS (dynamically nonrotating geocentric reference system) (Brumberg et al., 1996). Such a theory gives the explicit expressions in terms of TCG (Geocentric Coordinate Time) of three Euler angles relating a DGRS to the ITRS (International Terrestrial Reference System). These angular quantities together with their TCG derivatives enable one to get all Earth's rotation parameters. At the same time, the analysis of observations result in the values for slightly different angles and their TCG derivatives characterizing the relationship between the ITRS and a KGRS (kinematically nonrotating geocentric reference system). The differences between these two sets of six quantities represent kinematical relativistic corrections (due to geodesic precession, geodesic nutation and luni-planetary terms). The paper presents these differences computed by means of the VSOP87 series (Bretagnon and Francou, 1988). In particular, in analysing observations at the microarcsecond level these expressions will permit an experimental check of geodesic precession in a more direct manner than it is done nowadays (Bertotti et al., 1987).
\end{abstract}

\section{Introduction}

The aim of this paper is to supply numerical values for the quantities involved in the relativistic barycenric and geocentric reference systems (RSs) defined in Brumberg et al.(1996) and Brumberg (1997a). Using the VSOP87 series for the motion of the major planets (Bretagnon and Francou, 1988) this work was started in Brumberg et al.(1992) by computing the expressions for the geodesic rotation vector and its derivative and in Bretagnon et al.(1997) by computing the differences of the Euler angles for the geocentric rotation matrix relating the dynamical and kinematical systems. Our aim is to compute the differences of the Euler angles derivatives as well. In anticipating the space astrometry projects of microarcsecond accuracy it enables one to consider completely the effect of the geodesic rotation on the Earth's rotation parameters. 


\section{Barycentric and geocentric RSs}

In dealing with RSs we use the same notation as in Brumberg et al.(1996) and Brumberg (1997a), i.e. B - barycentric, G - geocentric, V - VLBI, C - ecliptical, $\mathrm{Q}$ - equatorial, $\mathrm{D}$ - dynamical, $\mathrm{K}$ - kinematical, ${ }^{+}$- rotating. At the barycentric level we have three systems BRSV, BRSC and BRSQ with timescale $t=\mathrm{TCB}$ and spatial coordinates $x=\left(x^{i}\right), x_{C}=\left(x_{C}^{i}\right)$ and $x_{Q}=\left(x_{Q}^{i}\right)$, respectively. At the geocentric level each of these systems generates by means of the BRS $\rightarrow$ GRS two geocentric RSs, dynamically or kinematically nonrotating ones with respect to the generating BRS. As a result, there will be six geocentric RSs, i.e. GRSV, GRSC and GRSQ for D and K versions with timescale $u=$ TCG and spatial coordinates $w=\left(w^{i}\right), w_{C}=\left(w_{C}^{i}\right)$ and $w_{Q}=\left(w_{Q}^{i}\right)$, respectively. If necessary, we will distinguish $\mathrm{D}$ and $\mathrm{K}$ versions by writing $w^{i}$ with $q=1$ for version $\mathrm{D}$ and $q=$ 0 for version $\mathrm{K}$. One more geocentric system is $\mathrm{GRS}^{+}$rotating with the Earth and having spatial coordinates $y=\left(y^{i}\right)$. By identifying BRSV and GRS ${ }^{+}$with ICRS and ITRS, respectively, the problem is to determine all relationships between these systems enabling one to obtain an unambiguous relativistic interpretation of Earth's rotation parameters as well as related astronomical concepts.

At the barycentric level all three RSs are mutually related by the constant rotation matrices

$$
x_{C}=P_{C} x, \quad x_{Q}=P_{Q} x .
$$

Very approximately

$$
P_{Q}=E, \quad P_{C}=\left(\begin{array}{ccc}
1 & 0 & 0 \\
0 & \cos \varepsilon & \sin \varepsilon \\
0 & -\sin \varepsilon & \cos \varepsilon
\end{array}\right),
$$

where $E$ stands for the unit matrix and $\varepsilon$ is the mean obliquity. This constant rotation is conserved at the geocentric level in the relationships between $\mathrm{V}, \mathrm{C}$ and $\mathrm{Q}$ GRSs of the same type (with respect to $\mathrm{D}$ or $\mathrm{K}$ versions). The matrix $P_{C}$ may be determined from the comparison of the VSOP87 planetary theories (Bretagnon and Francou, 1988) with observations of the major planets within BRSV (ICRS) background. Matrix $P_{Q}$ is to be determined at the geocentric level by comparison of the Earth's rotation theory like SMART97 (Bretagnon et al., 1998) with observations within GRS ${ }^{+}$(ITRS) background (see below). The relationship with GRS ${ }^{+}$involves the Earth's rotation matrix $\hat{P}(u)$

$$
y=\underset{q}{\hat{P}}(u) \underset{q}{w_{C}}=\underset{q}{\hat{P}}(u) P_{C} \underset{q}{w}=\underset{q}{\hat{P}}(u) P_{C} P_{Q}^{T} \underset{q}{w_{Q}} \text {. }
$$

The Earth's rotation matrix may be expressed in terms of three Euler angles to be determined by some Earth rotation theory. Following the traditional choice by Tisserand (1891) and Smart (1953) we will use here

$$
\hat{P}(u)=D_{3}(\varphi) D_{1}(-\theta) D_{3}(-\psi), \quad \hat{P}(u)=D_{3}\left(\varphi_{K}\right) D_{1}\left(-\theta_{K}\right) D_{3}\left(-\psi_{K}\right),
$$

with elementary rotations

$$
D_{1}(\alpha)=\left(\begin{array}{ccc}
1 & 0 & 0 \\
0 & \cos \alpha & \sin \alpha \\
0 & -\sin \alpha & \cos \alpha
\end{array}\right), \quad D_{2}(\beta)=\left(\begin{array}{ccc}
\cos \beta & 0 & -\sin \beta \\
0 & 1 & 0 \\
\sin \beta & 0 & \cos \beta
\end{array}\right),
$$




$$
D_{3}(\gamma)=\left(\begin{array}{rcc}
\cos \gamma & \sin \gamma & 0 \\
-\sin \gamma & \cos \gamma & 0 \\
0 & 0 & 1
\end{array}\right) .
$$

The relationships between the $\mathrm{D}$ and $\mathrm{K}$ systems involve the geodesic rotation matrix $F$

$$
\underset{0}{w}=\left(E-c^{-2} F\right) \underset{1}{w}, \quad \underset{0}{w_{C}}=\left(E-c^{-2} F_{C}\right) \underset{1}{w_{C}}, \quad \underset{0}{w_{Q}}=\left(E-c^{-2} F_{Q}\right) \underset{1}{w_{Q}},
$$

and

$$
\hat{P}(u)=\underset{0}{P}(u)\left(E-c^{-2} F_{C}\right)
$$

with

$$
F_{C}=P_{C} F P_{C}^{T}, \quad F_{Q}=P_{Q} F P_{Q}^{T} .
$$

Instead of rotation matrix $F$ one may consider its equivalent vector representation

$$
F^{i j}=\varepsilon_{i j k} F^{k}, \quad \varepsilon_{i j k}=\frac{1}{2}(i-j)(j-k)(k-i),
$$

so that the matrix product in (2.6) may be reduced to

$$
F w=-\varepsilon_{i j k} F^{j} w^{k} .
$$

The components of $F_{C}^{k}$ and $\dot{F}_{C}^{k}$ have been evaluated in Brumberg et al.(1992).

The relationships between barycentric and geocentric RSs are based on the $B R S \rightarrow$ GRS transformation (Brumberg, 1995 and references therein)

$$
\begin{gathered}
u=t-c^{-2}\left[A(t)+v_{E}^{k} r_{E}^{k}\right]+\ldots, \\
w^{i}=r_{E}^{i}+c^{-2}\left\{\left[\frac{1}{2} v_{E}^{i} v_{E}^{k}+q F^{i k}(t)+D^{i k}(t)\right] r_{E}^{k}+D^{i k m}(t) r_{E}^{k} r_{E}^{m}\right\}+\ldots,
\end{gathered}
$$

with

$$
\begin{gathered}
r_{E}^{i}=x^{i}-x_{E}^{i}(t), \quad v_{E}^{i}=\dot{x}_{E}^{i}(t), \\
\dot{A}(t)=\frac{1}{2} v_{E}^{2}+\bar{U}_{E}\left(\mathbf{x}_{E}\right)
\end{gathered}
$$

and

$$
D^{i k}(t)=\delta_{i k} \bar{U}_{E}\left(\mathbf{x}_{E}\right), \quad D^{i j k}(t)=\frac{1}{2}\left(\delta_{i j} a_{E}^{k}+\delta_{i k} a_{E}^{j}-\delta_{j k} a_{E}^{i}\right),
$$

where $\bar{U}_{E}(\mathbf{x})$ stands for the Newtonian potential of all solar system bodies except the Earth, $x_{E}^{i}, v_{E}^{i}$ and $a_{E}^{i}$ being Earth's BRS position, velocity and acceleration, respectively. This transformation is written for $\mathrm{V}$ versions of RSs. For $\mathrm{C}$ or $\mathrm{Q}$ versions one should convert all $\mathrm{V}$ quantities to $\mathrm{C}$ or $\mathrm{Q}$ quantities, respectively.

Let $t^{*}$ be the BRS moment of time corresponding to an event with the GRS coordinates $\left(u, w^{i}=0\right)$ (Klioner and Voinov, 1993). Then $u$ and $t^{*}$ are related by the time equation

Hence,

$$
u=t^{*}-c^{-2} A\left(t^{*}\right)+\ldots
$$


Expanding the right-hand member of (2.12) in the vicinity of $t^{*}$ one gets the inverse GRS $\rightarrow$ BRS transformation

$$
\begin{gathered}
t=u+c^{-2}\left[A(u)+z_{E}^{k} w^{k}\right]+\ldots, \\
x^{i}=w^{i}+z_{E}^{i}(u)+c^{-2}\left[\left(\frac{1}{2} v_{E}^{i} v_{E}^{k}-q F^{i k}-D^{i k}\right) w^{k}-D^{i k m} w^{k} w^{m}\right]+\ldots,
\end{gathered}
$$

with functions $z_{E}^{i}=z_{E}^{i}(u)$ characterizing the motion of the geocenter in terms of TCG and determined by

$$
z_{E}^{i}(u)=x_{E}^{i}\left(t^{*}\right)
$$

This inverse transformation is used, for example, to transform GRS space-time coordinates of terrestrial ground stations into their BRS space-time coordinates.

\section{K-D for Euler angles}

The first of relations (2.3) for the dynamical version $q=1$ represents a relationship between GRS ${ }^{+}$(ITRS) and DGRSC. The Euler angles $\varphi, \psi$ and $\theta$ together with their TCG derivatives result from the solution of the Earth's rotation equations written in the DGRSC. This solution may be thought of as consisting of two parts, a Newtonian one corresponding to the formal Newtonian equations of the Earth's rotation, and relativistic correction terms generated by the relativistic contributions to the right-hand sides of the Earth's rotation equations. These dynamical corrections $\delta \varphi, \delta \psi$ and $\delta \theta$ as well as their TCG derivatives are neglected here due to their small size in the DGRSC (Brumberg, 1997b, Klioner, 1997, and references therein). For the Newtonian solution of the Earth's rotation problem we choose here the SMART97 theory (Bretagnon et al., 1998). On the other hand astronomical observations of the Earth's rotation may be interpreted as performed in KGRSC resulting in the angles $\varphi_{K}, \psi_{K}$ and $\theta_{K}$ and their TCG derivatives. They differ from their dynamical counterparts due to the geodesic rotation vector $F^{K}$. Using (2.4) and (2.7) it follows that

$$
\begin{gathered}
\varphi-\varphi_{K}=-\frac{c^{-2}}{\sin \theta}\left(F_{C}^{1} \sin \psi+F_{C}^{2} \cos \psi\right), \\
\theta-\theta_{K}=c^{-2}\left(F_{C}^{1} \cos \psi-F_{C}^{2} \sin \psi\right),
\end{gathered}
$$

and

$$
\psi-\psi_{K}=c^{-2}\left[F_{C}^{3}-\frac{\cos \theta}{\sin \theta}\left(F_{C}^{1} \sin \psi+F_{C}^{2} \cos \psi\right)\right] .
$$

In this way the matrix $\hat{P}(u)$ is known (for both $\mathrm{D}$ and $\mathrm{K}$ versions) from the Earth rotation theory. Having found the constant matrix $P_{C}$ from the planetary motions one may determined the relationship between GRS* and GRSV from the second of relations (2.3). The main plane of GRS ${ }^{+}$is the equator of date. Having determined the mutual orientation of GRS ${ }^{+}$and GRSV one may find the constant matrix $P_{Q}$ defining the main plane of GRSQ as a fixed plane for some definite epoch. The inverse transformation GRSQ $\rightarrow$ BRSQ enables one to fix the BRSQ. 
Expressions (3.1)-(3.3) were evaluated first in (Bretagnon et al., 1997) using the series of SMART97. The three main RSs considered in (Bretagnon et al., 1997) are

$$
\mathrm{O} x y z=\mathrm{GRSC}, \quad \mathrm{O} \xi \eta \zeta=\mathrm{GRS}^{+}, \quad \text { O } \tilde{\xi} \tilde{\eta} \tilde{\zeta}=D_{3}(\alpha) \mathrm{GRS}^{+},
$$

$\alpha$ being the longitude of the axis associated with the principal moment of inertia of the Earth. This means that the system $0 \tilde{\xi} \tilde{\eta} \tilde{\zeta}$ is a terrestrial system with principal axes of inertia. Therefore, Bretagnon et al.(1997) angles $\omega$ and $\psi$ are, respectively, $-\theta$ and $-\psi$ of Brumberg et al.(1996) and Brumberg (1997a) whereas angle $\varphi$ has the same meaning in these papers. The expression for $\varphi_{K}-\varphi$ given in Bretagnon et al.(1997) should be taken with the opposite sign.

\section{K-D for Euler angles derivatives}

To evaluate the difference in the values of the Earth's rotation parameters (ERP) in dynamical and kinematical RS one should get along with $\varphi-\varphi_{K}, \psi-\psi_{K}$ and $\theta-\theta_{K}$ their derivatives $\dot{\varphi}-\dot{\varphi}_{K}, \dot{\psi}-\dot{\psi}_{K}$ and $\dot{\theta}-\dot{\theta}_{K}$. They may be computed by means of formulas

$$
\begin{gathered}
\dot{\varphi}-\dot{\varphi}_{K}=-\frac{c^{-2}}{\sin \theta}\left[\dot{F}_{C}^{1} \sin \psi+\dot{F}_{C}^{2} \cos \psi+\left(\theta-\theta_{K}\right) \dot{\psi}+\left(\varphi-\varphi_{K}\right) \dot{\theta} \cos \theta\right], \\
\dot{\psi}-\dot{\psi}_{K}=c^{-2} \dot{F}_{C}^{3}-\frac{c^{-2}}{\sin \theta}\left[\left(\dot{F}_{C}^{1} \sin \psi+\dot{F}_{C}^{2} \cos \psi\right) \cos \theta+\left(\theta-\theta_{K}\right) \dot{\psi} \cos \theta+\left(\varphi-\varphi_{K}\right) \dot{\theta}\right],
\end{gathered}
$$

and

$$
\dot{\theta}-\dot{\theta}_{K}=c^{-2}\left[\dot{F}_{C}^{1} \cos \psi-\dot{F}_{C}^{2} \sin \psi+\left(\varphi-\varphi_{K}\right) \dot{\psi} \sin \theta\right],
$$

using (3.1)-(3.3) and SMART97 theory (Bretagnon et al., 1998) for the angles $\psi, \theta$ and their derivatives. The initial terms of the series for (4.1)-(4.3) are given in the Appendix.

\section{Earth's rotation parameters}

Representation (2.4) means splitting $P(u)$ into matrix $S$ of the diurnal rotation and matrix $N$ of the precession and nutation, i.e.

$$
P(u)=S N, \quad S=D_{3}(\varphi), \quad N=D_{1}(-\theta) D_{3}(-\psi)
$$

(ignoring for simplicity the motion of the Earth's poles). This splitting involves the representation of the total Earth's rotation velocity $\omega^{i}$ (GRSC components of the angular velocity of rotation of $\mathrm{GRS}^{+}$with respect to GRSC) in two parts

$$
\omega^{i}=\omega_{N}^{i}+N_{j i} \omega_{S}^{j}
$$

with

$$
\omega_{S}=\left(\begin{array}{c}
0 \\
0 \\
\dot{\varphi}
\end{array}\right), \quad \omega_{N}=\left(\begin{array}{c}
-\dot{\theta} \cos \psi \\
\dot{\theta} \sin \psi \\
-\dot{\psi}
\end{array}\right)
$$


By applying these relations to the $D$ and $K$ versions one gets

$$
\omega_{1}-\omega_{0} S=\left(\begin{array}{c}
0 \\
0 \\
\dot{\varphi}-\dot{\varphi}_{K}
\end{array}\right)
$$

and

$$
\omega_{1}{ }_{1}-\omega_{0}=\left(\begin{array}{c}
\left(\psi-\psi_{K}\right) \dot{\theta} \sin \psi-\left(\dot{\theta}-\dot{\theta}_{K}\right) \cos \psi \\
\left(\psi-\psi_{K}\right) \dot{\theta} \cos \psi+\left(\dot{\theta}-\dot{\theta}_{K}\right) \sin \psi \\
-\left(\dot{\psi}-\dot{\psi}_{K}\right)
\end{array}\right) .
$$

Relation (4.4) characterizes the difference of the determination of the sidereal time in dynamical and kinematical systems. Relation (4.5) gives the difference of precession-nutation contributions in the Earth's angular velocity in dynamical and kinematical systems. The initial terms of the series representing the two first rows of matrix (4.5) are reproduced in the Appendix.

\section{Conclusion}

Using VSOP87 planetary theories and SMART97 Earth rotation theory we have computed the relativistic kinematical differences in the Euler angles and their TCG derivatives relating the ITRS and the geocentric ecliptical reference system GRSC in dynamical and kinematical versions. For future observations at the microarcsecond level it enables one to consider more rigorously the Earth's rotation parameters in different reference systems. For the present analysis it was sufficient for us at the barycentric level to relate the ICRS with equatorial and ecliptical reference systems just by means of simple matrices (2.2). The initial terms of the series determined here are given in the Appendix. The complete series are available by request to the second author.

In view of the present analysis it is interesting to consider the possibility of checking geodesic precession directly by comparing ERP referred to DGRSC and KGRSC. Geodesic precession has been tested only implicitly by analyzing the lunar perigee motion with the aid of LLR and VLBI observations (Bertotti et al., 1987).

Acknowledgments. The first author appreciates the support of the Russian Foundation of Fundamental Researches (Grant No. 99-02-16793).

\section{References}

Bertotti, B., Ciufolini, I., and Bender, P.L., 1987, Phys. Rev. Lett., 58, 1062.

Bretagnon, P. and Francou, G., 1988, Astron. Astrophys., 202, 309.

Bretagnon, P., Rocher, P., and Simon, J.-L., 1997, Astron. Astrophys., 319, 305.

Bretagnon, P., Francou, G., Rocher, P., and Simon, J.-L., 1998, Astron. Astrophys, , 329, 329.

Brumberg, V.A., 1995, J. of Geodynamics, 20, 181. 
Brumberg, V.A., 1997a, in Dynamics and Astrometry of Natural and Artificial Celestial Bodies (IAU Colloquium No. 165, Poznan, 1996, eds. I.M. Wytrzyszczak, J.H. Lieske and R.A. Feldman), Kluwer, 439.

Brumberg, V.A., 1997b, Notes Sci. et Tech. du BDL, S057, Paris, 1.

Brumberg, V.A., Bretagnon, P., and Francou, G., 1992, Journées 1991, ed. N. Capitaine, Obs. de Paris, 141.

Brumberg, V.A., Bretagnon, P., and Guinot, B., 1996, Celest. Mech., 64, 231.

Klioner, S.A., 1997, in Dynamics and Astrometry of Natural and Artificial Celestial Bodies (IAU Colloquium No. 165, Poznan, 1996, eds. I.M. Wytrzyszczak, J.H. Lieske and R.A. Feldman), Kluwer, 383.

Klioner, S.A. and Voinov, A.V., 1993, Phys. Rev. D, 48, 1451.

Smart, W.M., 1953, Celestial Mechanics, Longmans, London

Tisserand, F., 1891, Traité de Mécanique Céleste, Gauthier-Villars, Paris, t. II

\section{Appendix}

We reproduce below the initial terms of the series for $\varphi-\varphi_{K}, \psi-\psi_{K}, \theta-\theta_{K}$, $\dot{\varphi}-\dot{\varphi}_{K}, \dot{\psi}-\dot{\psi}_{K}, \dot{\theta}-\dot{\theta}_{K}$ and first two rows of matrix (4.5). The time $t$ is measured in Julian years from J2000. These series involve 12 angular variables, i.e. 8 mean planetary longitudes with respect to the fixed equinox J2000 $\lambda_{i}(1 \leq i \leq 8)$, the Delaunay arguments $D, F, l$ of the Moon and the mean angle of rotation of the Earth $\bar{\varphi}$. These arguments expressed in radians are represented by linear functions of time as follows:

$$
\begin{aligned}
\lambda_{1} & =4.40260867435+26087.9031415742 t \\
\lambda_{2} & =3.17614652884+10213.2855462110 t \\
\lambda_{3} & =1.75347029148+6283.0758511455 t \\
\lambda_{4} & =6.20347594486+3340.6124266998 t \\
\lambda_{5} & =0.59954632934+529.6909650946 t \\
\lambda_{6} & =0.87401658845+213.2990954380 t \\
\lambda_{7} & =5.48129370354+74.7815985673 t \\
\lambda_{8} & =5.31188611871+38.1330356378 t \\
D & =5.19846640063+77713.7714481804 t \\
F & =1.62790513602+84334.6615717837 t \\
l & =2.35555563875+83286.9142477147 t \\
\bar{\varphi} & =4.89496121282+2301216.7536515365 t .
\end{aligned}
$$

These values are slightly different from those given in (Bretagnon et al., 1998). The following three series representing the differences $\varphi-\varphi_{K}, \psi-\psi_{K}, \theta-\theta_{K}$ are reproduced here with some more terms as compared with (Bretagnon et al., 1997), their coefficients being expressed in $\mu$ as:

$$
\begin{aligned}
\varphi & -\varphi_{K}=-2.99 t-54771.03 t^{2}-802.06 t^{3}+ \\
& +3.28 \sin \left(\lambda_{3}+D-F\right)-0.24 \sin \left(2 \lambda_{5}-5 \lambda_{6}\right)-0.36 \cos \left(2 \lambda_{5}-5 \lambda_{6}\right)+ \\
& +0.07 \sin \left(8 \lambda_{2}-13 \lambda_{3}\right)-0.04 \cos \left(8 \lambda_{2}-13 \lambda_{3}\right)+0.02 \sin \left(3 \lambda_{2}-5 \lambda_{3}\right)+
\end{aligned}
$$


$+0.07 \cos \left(3 \lambda_{2}-5 \lambda_{3}\right)-0.05 \sin \left(\lambda_{3}+F\right)-0.04 \sin \left(\lambda_{3}-F\right)+$

$+0.03 \cos 2 \lambda_{5}+0.02 \cos 2 \lambda_{6}+0.02 \cos \lambda_{5}-0.01 \cos \left(\lambda_{3}+\lambda_{5}\right)+$

$+t\left[0.20 \sin \lambda_{3}+0.85 \cos \lambda_{3}+0.80 \cos \left(\lambda_{3}+D-F\right)-0.14 \sin \left(2 \lambda_{5}-5 \lambda_{6}\right)+\right.$

$+0.05 \cos \left(2 \lambda_{5}-5 \lambda_{6}\right)-0.02 \sin \left(8 \lambda_{2}-13 \lambda_{3}\right)-0.02 \cos \left(8 \lambda_{2}-13 \lambda_{3}\right)+$

$\left.+0.02 \sin \left(3 \lambda_{2}-5 \lambda_{3}\right)-0.01 \cos \left(\lambda_{3}+F\right)\right]+$

$+t^{2}\left[-0.51 \sin \left(\lambda_{3}+D-F\right)-5.64 \cos \left(\lambda_{3}+D-F\right)+0.03 \sin 2 \lambda_{3}+0.34 \cos 2 \lambda_{3}+\right.$

$+0.05 \sin \lambda_{3}-0.02 \cos \lambda_{3}+0.06 \cos \left(2 \lambda_{3}+2 D\right)-0.06 \cos \left(2 \lambda_{3}+2 D-2 F\right)+$

$\left.+0.02 \cos \left(2 \lambda_{5}-5 \lambda_{6}\right)+0.01 \sin 3 \lambda_{3}-0.01 \cos \left(\lambda_{3}+D+F\right)\right]+$

$+t^{3}\left[2.37 \sin \left(\lambda_{3}+D-F\right)-0.20 \cos \left(\lambda_{3}+D-F\right)-0.24 \sin 2 \lambda_{3}+0.02 \cos 2 \lambda_{3}-\right.$

- $\left.0.04 \sin \left(2 \lambda_{3}+2 D\right)+0.04 \sin \left(2 \lambda_{3}+2 D-2 F\right)-0.02 \cos \lambda_{3}\right]+\ldots$,

$\psi-\psi_{K}=19198827.44 t-50386.32 t^{2}-754.09 t^{3}-$

- $34.28 \sin \lambda_{3}-149.22 \cos \lambda_{3}+3.01 \sin \left(\lambda_{3}+D-F\right)-1.73 \sin 2 \lambda_{3}+$

$+0.84 \cos 2 \lambda_{3}-0.26 \sin \left(2 \lambda_{5}-5 \lambda_{6}\right)-0.33 \cos \left(2 \lambda_{5}-5 \lambda_{6}\right)-0.37 \sin D-$

- $0.05 \sin \left(4 \lambda_{3}-8 \lambda_{4}+3 \lambda_{5}\right)+0.17 \cos \left(4 \lambda_{3}-8 \lambda_{4}+3 \lambda_{5}\right)+0.21 \sin \left(\lambda_{3}-\lambda_{5}\right)-$

- $0.13 \sin \left(2 \lambda_{2}-2 \lambda_{3}\right)+0.09 \sin \left(\lambda_{2}-\lambda_{3}\right)+0.09 \sin \left(8 \lambda_{2}-13 \lambda_{3}\right)+$

$+t\left[-7.36 \sin \lambda_{3}+6.47 \cos \lambda_{3}+0.73 \cos \left(\lambda_{3}+D-F\right)+0.19 \sin 2 \lambda_{3}+\right.$

$+0.15 \cos 2 \lambda_{3}-0.13 \sin \left(2 \lambda_{5}-5 \lambda_{6}\right)+0.04 \cos \left(2 \lambda_{5}-5 \lambda_{6}\right)-$

$-0.02 \sin \left(8 \lambda_{2}-13 \lambda_{3}\right)-0.03 \cos \left(8 \lambda_{2}-13 \lambda_{3}\right)+0.02 \sin \left(3 \lambda_{2}-5 \lambda_{3}\right)-$

- $\left.0.02 \sin \left(4 \lambda_{3}-8 \lambda_{4}+3 \lambda_{5}\right)-0.01 \cos \left(\lambda_{3}+F\right)\right]+$

$+t^{2}\left[-0.47 \sin \left(\lambda_{3}+D-F\right)-6.15 \cos \left(\lambda_{3}+D-F\right)+0.30 \sin \lambda_{3}+0.29 \cos \lambda_{3}+\right.$

$+0.04 \sin 2 \lambda_{3}+0.35 \cos 2 \lambda_{3}+0.06 \cos \left(2 \lambda_{3}+2 D\right)-0.06 \cos \left(2 \lambda_{3}+2 D-2 F\right)+$

$\left.+0.01 \cos \left(2 \lambda_{5}-5 \lambda_{6}\right)+0.01 \sin 3 \lambda_{3}-0.01 \cos \left(\lambda_{3}+D+F\right)\right]+$

$+t^{3}\left[2.41 \sin \left(\lambda_{3}+D-F\right)-0.20 \cos \left(\lambda_{3}+D-F\right)-0.25 \sin 2 \lambda_{3}+0.02 \cos 2 \lambda_{3}-\right.$

- $\left.0.04 \sin \left(2 \lambda_{3}+2 D\right)+0.04 \sin \left(2 \lambda_{3}+2 D-2 F\right)-0.03 \cos \lambda_{3}\right]+\ldots$,

$$
\begin{aligned}
\theta & -\theta_{K}=9.55 t+1954.11 t^{2}-4721.80 t^{3}- \\
& -1.30 \cos \left(\lambda_{3}+D-F\right)+0.17 \sin \left(2 \lambda_{5}-5 \lambda_{6}\right)-0.09 \cos \left(2 \lambda_{5}-5 \lambda_{6}\right)+ \\
& +0.02 \sin \left(8 \lambda_{2}-13 \lambda_{3}\right)+0.03 \cos \left(8 \lambda_{2}-13 \lambda_{3}\right)-0.03 \sin \left(3 \lambda_{2}-5 \lambda_{3}\right)+ \\
& +0.02 \cos \left(\lambda_{3}+F\right)+0.02 \cos \left(\lambda_{3}-F\right)+0.01 \sin 2 \lambda_{5}+ \\
& +t\left[0.32 \sin \left(\lambda_{3}+D-F\right)-0.03 \sin \left(2 \lambda_{5}-5 \lambda_{6}\right)-0.04 \cos \left(2 \lambda_{5}-5 \lambda_{6}\right)-\right. \\
& \left.-0.03 \cos \lambda_{3}\right]+ \\
& +t^{2}\left[-1.82 \sin \left(\lambda_{3}+D-F\right)+0.04 \cos \left(\lambda_{3}+D-F\right)+0.14 \sin 2 \lambda_{3}+0.02 \sin \lambda_{3}+\right. \\
& \left.+0.08 \cos \lambda_{3}+0.02 \sin \left(2 \lambda_{3}+2 D\right)-0.02 \sin \left(2 \lambda_{3}+2 D-2 F\right)\right]+ \\
& +t^{3}\left[-0.04 \sin \left(\lambda_{3}+D-F\right)-0.45 \cos \left(\lambda_{3}+D-F\right)+0.07 \cos 2 \lambda_{3}+\right. \\
& \left.+0.01 \cos \left(2 \lambda_{3}+2 D\right)-0.01 \cos \left(2 \lambda_{3}+2 D-2 F\right)\right]+\ldots
\end{aligned}
$$

The derivatives $\dot{\varphi}-\dot{\varphi}_{K}, \dot{\psi}-\dot{\psi}_{K}, \dot{\theta}-\dot{\theta}_{K}$ could be obtained by differentiating (A.1)-(A.3). But we give below their expressions resulting directly from (4.1)(4.3). Differentiation of (A.1)-(A.3) was used only for checking purposes. The 
series for the differences $\dot{\varphi}-\dot{\varphi}_{K}, \dot{\psi}-\dot{\psi}_{K}, \dot{\theta}-\dot{\theta}_{K}$ multiplied by $10^{6}$ are as follows:

$$
\begin{aligned}
10^{6}(\dot{\varphi} & \left.-\dot{\varphi}_{K}\right)=-0.53 t-0.01 t^{2}+0.03 t^{3}- \\
& -0.02 \cos \left(\lambda_{3}+F\right)+0.01 \cos \left(\lambda_{3}-F\right)- \\
& -0.03 t \sin \lambda_{3}- \\
& -t^{2}\left[0.05 \sin \left(2 \lambda_{3}+2 D\right)+0.02 \sin 2 \lambda_{3}\right]- \\
& -t^{3}\left[0.03 \cos \left(2 \lambda_{3}+2 D\right)+0.01 \cos 2 \lambda_{3}\right]+ \\
& +0.01 t^{4} \sin \left(2 \lambda_{3}+2 D\right)+\ldots,
\end{aligned}
$$

$$
\begin{aligned}
10^{6}(\dot{\psi} & \left.-\dot{\psi}_{K}\right)=93.08-0.49 t-0.01 t^{2}+0.02 t^{3}+ \\
& +4.55 \sin \lambda_{3}-1.04 \cos \lambda_{3}-0.05 \sin 2 \lambda_{3}-0.11 \cos 2 \lambda_{3}-0.14 \cos D- \\
& -0.02 \cos \left(\lambda_{3}+F\right)-0.02 \cos (D+l)+0.01 \cos \left(\lambda_{3}-F\right)+ \\
& +t\left[-0.20 \sin \lambda_{3}-0.22 \cos \lambda_{3}+0.01 \cos 2 \lambda_{3}\right]+ \\
& +t^{2}\left[-0.05 \sin \left(2 \lambda_{3}+2 D\right)-0.02 \sin 2 \lambda_{3}+0.01 \sin \left(\lambda_{3}+D+F\right)-\right. \\
& \left.-0.01 \sin \left(2 \lambda_{3}+2 D+l\right)-0.01 \sin \left(\lambda_{3}+D-F\right)\right]- \\
& -t^{3}\left[0.03 \cos \left(2 \lambda_{3}+2 D\right)+0.02 \cos 2 \lambda_{3}\right]+ \\
& +0.01 t^{4} \sin \left(2 \lambda_{3}+2 D\right)+\ldots \\
10^{6}\left(\dot{\theta}-\dot{\theta}_{K}\right) & =0.02 t-0.07 t^{2}+0.02 t^{2} \cos \left(2 \lambda_{3}+2 D\right)+\ldots
\end{aligned}
$$

Finally, we reproduce the series representing the first two rows of matrix (4.5) multiplied by $10^{6}$ :

$$
\begin{aligned}
& 10^{6}\left(\omega_{N}^{1}-\omega_{0}^{1}\right)=-0.02 t+0.07 t^{2}+ \\
& +t^{2}\left[-1.76 \sin \left(2 \lambda_{3}+2 D\right)-0.02 \cos \left(2 \lambda_{3}+2 D\right)-0.77 \sin 2 \lambda_{3}+\right. \\
& +0.36 \sin \left(\lambda_{3}+D+F\right)-0.35 \sin \left(2 \lambda_{3}+2 D+l\right)-0.34 \sin \left(\lambda_{3}+D-F\right)+ \\
& +0.07 \sin \left(\lambda_{3}+D+F+l\right)-0.07 \sin \left(2 \lambda_{3}+4 D-l\right)-0.06 \sin \left(2 \lambda_{3}+4 D\right)+ \\
& +0.04 \cos 3 \lambda_{3}-0.05 \sin \left(2 \lambda_{3}+2 D+2 l\right)+0.05 \sin \left(2 \lambda_{3}+2 D-l\right)- \\
& -0.03 \sin \left(\lambda_{3}+D-F+l\right)-0.03 \sin \left(\lambda_{3}+D-F-l\right)-0.01 \sin \left(2 \lambda_{3}+4 D+l\right)+ \\
& \left.+0.01 \sin \left(\lambda_{3}+3 D+F-l\right)+0.01 \sin \left(2 \lambda_{3}+l\right)+0.01 \sin \left(\lambda_{3}+3 D+F\right)\right]+ \\
& +t^{3}\left[0.03 \sin \left(2 \lambda_{3}+2 D\right)-0.86 \cos \left(2 \lambda_{3}+2 D\right)+0.01 \sin 2 \lambda_{3}-0.37 \cos 2 \lambda_{3}-\right. \\
& -0.17 \cos \left(2 \lambda_{3}+2 D+l\right)+0.09 \cos \left(\lambda_{3}+D+F\right)-0.08 \cos \left(\lambda_{3}+D-F\right)- \\
& -0.03 \cos \left(2 \lambda_{3}+4 D-l\right)-0.03 \cos \left(2 \lambda_{3}+4 D\right)-0.02 \cos \left(2 \lambda_{3}+2 D+2 l\right)+ \\
& \left.+0.02 \cos \left(2 \lambda_{3}+2 D-l\right)-0.02 \sin 3 \lambda_{3}+0.02 \cos \left(\lambda_{3}+D+F+l\right)\right]+ \\
& +t^{4}\left[0.23 \sin \left(2 \lambda_{3}+2 D\right)+0.02 \cos \left(2 \lambda_{3}+2 D\right)+0.10 \sin 2 \lambda_{3}+\right. \\
& \left.+0.05 \sin \left(2 \lambda_{3}+2 D+l\right)-0.01 \sin \left(\lambda_{3}+D+F\right)+0.01 \sin \left(\lambda_{3}+D-F\right)\right]+ \\
& +t^{5}\left[0.04 \cos \left(2 \lambda_{3}+2 D\right)+0.02 \cos 2 \lambda_{3}\right]+\ldots,
\end{aligned}
$$




$$
\begin{aligned}
& 10^{6}\left(\omega_{1}^{2}-\omega_{N}^{2}\right)=-0.03 t^{3}+ \\
& +t\left[-7.20 \sin \left(2 \lambda_{3}+2 D\right)-3.14 \sin \left(2 \lambda_{3}\right)+1.47 \sin \left(\lambda_{3}+D+F\right)-\right. \\
& -1.43 \sin \left(2 \lambda_{3}+2 D+l\right)-1.41 \sin \left(\lambda_{3}+D-F\right)+0.29 \sin \left(\lambda_{3}+D+F+l\right)- \\
& -0.27 \sin \left(2 \lambda_{3}+4 D-l\right)-0.24 \sin \left(2 \lambda_{3}+4 D\right)+0.04 \sin 3 \lambda_{3}+0.18 \cos 3 \lambda_{3}- \\
& -0.20 \sin \left(2 \lambda_{3}+2 D+2 l\right)+0.20 \sin \left(2 \lambda_{3}+2 D-l\right)-0.12 \sin \left(\lambda_{3}+D-F+l\right)- \\
& -0.11 \sin \left(\lambda_{3}+D-F-l\right)-0.06 \sin \left(2 \lambda_{3}+4 D+l\right)+0.06 \sin \left(\lambda_{3}+3 D+F-l\right)+ \\
& +0.05 \sin \left(2 \lambda_{3}+l\right)+0.05 \sin \left(\lambda_{3}+3 D+F\right)+0.03 \sin 2 \bar{\varphi}-0.02 \cos 2 \bar{\varphi}+ \\
& \left.+0.04 \sin \left(\lambda_{3}+D+F+2 l\right)-0.04 \sin \left(\lambda_{3}+D+F-l\right)\right]+ \\
& +t^{2}\left[0.06 \sin \left(2 \lambda_{3}+2 D\right)-3.51 \cos \left(2 \lambda_{3}+2 D\right)+0.02 \sin 2 \lambda_{3}-1.53 \cos 2 \lambda_{3}+\right. \\
& +0.01 \sin \left(2 \lambda_{3}+2 D+l\right)-0.70 \cos \left(2 \lambda_{3}+2 D+l\right)+0.36 \cos \left(\lambda_{3}+D+F\right)- \\
& -0.34 \cos \left(\lambda_{3}+D-F\right)-0.13 \cos \left(2 \lambda_{3}+4 D-l\right)-0.12 \cos \left(2 \lambda_{3}+4 D\right)- \\
& -0.10 \cos \left(2 \lambda_{3}+2 D+2 l\right)+0.10 \cos \left(2 \lambda_{3}+2 D-l\right)-0.08 \sin 3 \lambda_{3}+0.01 \cos 3 \lambda_{3}+ \\
& +0.07 \cos \left(\lambda_{3}+D+F+l\right)-0.03 \cos \left(2 \lambda_{3}+4 D+l\right)-0.03 \cos \left(\lambda_{3}+D-F+l\right)- \\
& -0.03 \cos \left(\lambda_{3}+D-F-l\right)+0.03 \cos \left(2 \lambda_{3}+l\right)-0.01 \sin \lambda_{3}+0.01 \sin \left(\lambda_{3}+2 D\right)- \\
& \left.-0.01 \cos \left(2 \lambda_{3}+2 D-2 F\right)+0.01 \cos \left(\lambda_{3}+3 D+F-l\right)\right]+ \\
& +t^{3}\left[1.07 \sin \left(2 \lambda_{3}+2 D\right)+0.08 \cos \left(2 \lambda_{3}+2 D\right)+0.47 \sin 2 \lambda_{3}+0.01 \cos 2 \lambda_{3}+\right. \\
& +0.21 \sin \left(2 \lambda_{3}+2 D+l\right)-0.09 \sin \left(\lambda_{3}+D+F\right)-0.02 \cos \left(\lambda_{3}+D+F\right)+ \\
& +0.08 \sin \left(\lambda_{3}+D-F\right)+0.04 \sin \left(2 \lambda_{3}+4 D-l\right)+0.04 \sin \left(2 \lambda_{3}+4 D\right)- \\
& -0.03 \sin \left(2 \lambda_{3}+2 D-l\right)+0.03 \sin \left(2 \lambda_{3}+2 D+2 l\right)-0.02 \cos 3 \lambda_{3}- \\
& \left.-0.02 \sin \left(\lambda_{3}+D+F+l\right)\right]+ \\
& +t^{4}\left[-0.04 \sin \left(2 \lambda_{3}+2 D\right)+0.24 \cos \left(2 \lambda_{3}+2 D\right)+0.11 \cos 2 \lambda_{3}+\right. \\
& \left.+0.05 \cos \left(2 \lambda_{3}+2 D+l\right)-0.01 \cos \left(\lambda_{3}+D+F\right)+0.01 \cos \left(\lambda_{3}+D-F\right)\right]- \\
& -t^{5}\left[0.04 \sin \left(2 \lambda_{3}+2 D\right)+0.01 \cos \left(2 \lambda_{3}+2 D\right)+0.02 \sin 2 \lambda_{3}\right]+\ldots \\
& +0.7)
\end{aligned}
$$

The coefficients of the series (A.4)-(A.8) are expressed in radians. 